\title{
A GENERALIZED HANKEL TRANSFORM AND ITS USE FOR SOLVING CERTAIN PARTIAL DIFFERENTIAL EQUATIONS
}

\author{
I. ALI ${ }^{1}$ and S. KALLA ${ }^{2}$
}

(Received 11 July 1996; revised 14 August 1997)

\begin{abstract}
We introduce a generalized form of the Hankel transform, and study some of its properties. A partial differential equation associated with the problem of transport of a heavy pollutant (dust) from the ground level sources within the framework of the diffusion theory is treated by this integral transform. The pollutant concentration is expressed in terms of a given flux of dust from the ground surface to the atmosphere. Some special cases are derived.
\end{abstract}

\section{Introduction}

Integral transforms: Let $f(t)$ be a given function defined on an interval $(a, b)$ and belonging to a certain class of functions. An integral transform of $f(t)$ is a mapping of the form

$$
T[f(t) ; s]=\bar{f}(s)=\int_{a}^{b} K(s, t) f(t) d t,
$$

provided the integral exists; $K(s, t)$ is a prescribed function called the kernel of the transform $[5,11,12]$. Among the well-known transforms are the Laplace, Mellin, Stieltjes and Hankel transforms. The Laplace transform has been more widely used and is particularly suitable for problems governed by ordinary differential equations as well as problems in the theory of heat conduction. On the other hand, for problems in which there is axial symmetry and the radial variable ranges from 0 to $\infty$, the Hankel transform is found to be most appropriate. It is defined as

$$
H_{v}[f(z) ; s]=\tilde{f_{v}}(s)=\int_{0}^{\infty} z f(z) J_{v}(s z) d z,
$$

\footnotetext{
'Department of Mathematics and Computer Science, Kuwait University, P.O. Box 5969 Safat, Kuwait 13060

${ }^{2}$ CIMA, Facultad de Ingenería, Universidad del Zulia, Apartado - 10182, Maracaibo, Venezuela

(C) Australian Mathematical Society 1999, Serial-fee code 0334-2700/99
} 
where $J_{v}(z)$ is the usual Bessel function of first kind of order $v[8,13]$.

In the application of the Hankel transforms to physical problems, it is useful to have available some formulae connecting the Hankel transforms of derivatives of functions. For example,

$$
H_{\nu}\left[f_{z z}+\frac{1}{z} f_{z}-\frac{\nu^{2}}{z^{2}} f\right]=-s^{2} \overline{f_{v}}(s),
$$

provided both $z f(z)$ and $z f^{\prime}(z)$ tend to zero as $z \rightarrow 0, \infty$.

The diffusion of dust problem: Studies concerning environmental protection have lead investigators to tackle important problems dealing with the transport of pollutants in the atmosphere. A number of factors are involved in this phenomenon: weather conditions, the type of source and the properties of the pollutant. Initial studies were concerned with the transport of pollutants from industrial sources elevated from the ground level [3] and a technique for measuring air pollution from such sources was developed. However, the mathematical models governing the transport of pollutants from the ground-level sources have been defined primarily for cases of light particles. It is therefore of great interest to investigate the entrainment and transport of heavy particles (those with an intrinsic settling velocity such as dust of natural and industrial origin) into the atmosphere. There are many sources of such pollutants such as mine-waste, dumps, outdoor industrial yards, production areas and sand storms.

Many people have investigated the question of entrainment of heavy particles from the ground surface on which they lie and their transport in flow. Thus a theory of a particle-carrying heavy flow was developed by Barenblatt [2]. He showed that if the flow carries fairly heavy particles (or, in the case of lighter particles, if their concentrations in the flow are high), the flow turbulence energy is significantly suppressed, because some energy is expended on stripping the particles off the underlying surface, and on maintaining them in the suspended state. In this case one must analyze jointly the transport of the particles in the flow, and the flow itself .

The flow structure remains virtually unchanged by the presence of particles in it if the work expended by the flow on keeping the particles in suspension is small. In this case the transport of the particles in the flow can be treated within the framework of the diffusion theory.

The inequality $w / \alpha v_{*}<1$ was presented by Barenblatt $[1,2]$ as a criterion for the extended residence of particles in the flow where $\nu_{*}$ is the friction velocity, $w$ is the particle settling velocity, and $\alpha$ is the von Kármán constant. Byutner [4] conjectured that when $w / \alpha \nu_{*}>1$, the motion of the particles in the flow could be described by a series of up and down jumps. This type of motion is called saltation, which is the main mechanism of particle transport in sand storms.

Nowadays it is a common practice to solve complicated problems of atmospheric 
diffusion numerically, since numerical techniques admit a vast range of coefficients of equations, and boundary conditions that describe a given process. Nevertheless, analytical methods have their own merit, owing to their clarity and a global view of the development of the solution.

In this paper, we first introduce a generalized form of the Hankel transform and study some of its basic properties including the transform of a modified differential operator. Then we deal with the problem of transport of a heavy pollutant (dust) from a ground level aerial source within the framework of diffusion theory. An analytical solution of the problem is obtained by an appeal to the generalized Hankel transform. Different cases of the positive flow of dust to the atmosphere are considered, and some examples are given. The pollutant concentration is expressed in terms of known functions which can be tabulated easily.

\section{The generalized Hankel transform}

We define the generalized Hankel transform as

$$
H_{v}[f(x, z) ; s, a, c]=\int_{0}^{\infty} z^{a} f(x, z) J_{\nu}\left(s z^{c}\right) d z=\overline{f_{v}}(x, s ; a, c)=\overline{f_{v}}(s),
$$

where $\nu, s, a$, and $c$ are real numbers, and $f(x, z)$ belongs to a certain class of functions for which the integral exists. It is not difficult to establish the corresponding inversion formula by virtue of the Hankel inversion theorem [7], that is,

$$
f(x, z)=c z^{2 c-a-1} \int_{0}^{\infty} s \bar{f}_{\nu}(x, s ; a, c) J_{\nu}\left(s z^{c}\right) d s, \quad c>0 .
$$

For $a=c=1$ we have [11]

$$
H_{v}[f(x, z) ; s, 1,1]=\int_{0}^{\infty} z f(x, z) J_{v}(s z) d z=\overline{f_{v}}(s),
$$

and

$$
f(x, z)=\int_{0}^{\infty} s \overline{f_{v}}(s) J_{v}(s z) d s .
$$

Parseval type results: Let

$$
\overline{f_{v}}(x, s ; a, c)=\int_{0}^{\infty} z^{a} f(x, z) J_{v}\left(s z^{c}\right) d z
$$

and

$$
\bar{g}_{v}(x, s ; a, c)=\int_{0}^{\infty} z^{a} g(x, z) J_{v}\left(s z^{c}\right) d z
$$


where $f$ and $g$ are absolutely integrable functions on $\mathbf{R}$. Then we have

$$
\int_{0}^{\infty} z^{a} f(x, z) \bar{g}_{\nu}(x, s ; a, c) J_{\nu}\left(s z^{c}\right) d z=\int_{0}^{\infty} z^{a} g(x, z) \overline{f_{\nu}}(x, s ; a, c) J_{\nu}\left(s z^{c}\right) d z,
$$

and

$$
\int_{0}^{\infty} s \bar{g}_{\nu}(x, s ; a, c) \bar{f}_{\nu}(x, s ; a, c) d s=\frac{1}{c} \int_{0}^{\infty} z^{2(a-c)+1} g(x, z) f(x, z) d z
$$

Elementary properties of the generalized Hankel transform: A simple change of variables in the defining integral leads to

$$
H_{v}[f(x, \rho z) ; s, a, c]=\frac{1}{\rho^{a+1}} H_{v}\left[f(x, z) ; \frac{s}{\rho^{c}}, a, c\right],
$$

while another simple relation is obtained by making use of the recurrence relation

$$
J_{v-1}(x)-\frac{2 v}{x} J_{v}(x)+J_{v+1}(x)=0
$$

in the following form:

$$
H_{\nu}\left[z^{-c} f(x, z) ; s, a, c\right]=\frac{s}{2 v}\left\{H_{\nu-1}[f(x, z) ; s, a, c]+H_{v+1}[f(x, z) ; s, a, c]\right\} .
$$

Differential properties: The generalized Hankel transform of derivatives of a given function $f(x, z)$ are found to be

$$
\begin{aligned}
& H_{v}\left[f_{z}(x, z) ; s, a, c\right] \\
& =(v c-a) H_{v}\left[\frac{1}{z} f(x, z) ; s, a, c\right]-s c H_{v-1}\left[z^{c-1} f(x, z) ; s, a, c\right], \\
& H_{v}\left[f_{z z}(x, z) ; s, a, c\right] \\
& =\frac{s^{2}}{4 v}\left[\frac{(a+v c)(a+v c-1)}{v-1} H_{v-2}\left[z^{c-1} f(x, z) ; s, a+c-1, c\right]\right. \\
& -\left\{\frac{(a+\nu c)(\nu c-2 c-a+1)}{v-1}+\frac{(\nu c-a)(\nu c+2 c+a-1)}{v+1}\right\} \\
& \times H_{v}\left[z^{c-1} f(x, z) ; s, a+c-1, c\right] \\
& \left.+\frac{(\nu c-a)(v c-a+1)}{\nu+1} H_{v+2}\left[z^{c-1} f(x, z) ; s, a+c-1, c\right]\right],
\end{aligned}
$$

where $z^{a} f_{z}(x, z)$ and $z^{a} f(x, z)$ tend to zero as $z \rightarrow 0, \infty$. Let us define the auxiliary operator

$$
Q=\frac{\lambda}{z^{m-1}} \frac{\partial^{2}}{\partial z^{2}}+\frac{\rho}{z^{m}} \frac{\partial}{\partial z}-\frac{\lambda}{z^{m+1}}\left[c^{2} v^{2}-a^{2}+m\left(m-1+\frac{\rho}{\lambda}\right)\right],
$$


where $\lambda$ and $\rho$ are constants such that $\rho / \lambda=2(a-m)+1$. Such operators are useful when dealing with a number of boundary value problems. The generalized Hankel transform of the operator $Q$ is

$$
H_{v}[Q f ; s, a, c]=\Lambda-\lambda c^{2} s^{2} H_{\nu}\left[2^{2 c-m-1} f ; s, a, c\right],
$$

where

$$
\Lambda=\left.z^{a-m}\left(\lambda z \frac{\partial f}{\partial z}+\rho f\right) J_{\nu}\left(s z^{c}\right)\right|_{0} ^{\infty}-\left.\lambda f \frac{\partial}{\partial z}\left(z^{a-m+1} J_{\nu}\left(s z^{c}\right)\right)\right|_{0} ^{\infty} .
$$

The conditions on $f$ and $f_{z}$ when $z \rightarrow 0, \infty$ depend on the particular problem.

\section{Proofs:}

1) Integrating $H_{v}\left[f_{z}(x, z) ; s, a, c\right]=\int_{0}^{\infty} z^{a} f_{z}(x, z) J_{v}\left(s z^{c}\right) d z$ by parts and noting that [13]

$$
\frac{d}{d z}\left(J_{v}\left(s z^{c}\right)\right)=c s z^{c-1} J_{v-1}\left(s z^{c}\right)-\frac{c v}{z} J_{v}\left(s z^{c}\right),
$$

we obtain

$$
\begin{aligned}
& H_{v}\left[f_{z}(x, z) ; s, a, c\right] \\
& \quad=\left.z^{a} f(x, z) J_{v}\left(s z^{c}\right)\right|_{0} ^{\infty}-\int_{0}^{\infty} f(x, z)\left[a z^{a-1} J_{v}\left(s z^{c}\right)+z^{a} \frac{d}{d z}\left(J_{v}\left(s z^{c}\right)\right)\right] d z \\
& \quad=(c v-a) H_{v}\left[\frac{1}{z} f(x, z) ; s, a, c\right]-c s H_{v-1}\left[z^{c-1} f(x, z) ; s, a, c\right],
\end{aligned}
$$

which proves (10).

2) To obtain (12) we use (1) to find the generalized Hankel transform of the operator

$$
\frac{\lambda}{z^{m-1}} \frac{\partial^{2} f}{\partial z^{2}}+\frac{\rho}{z^{m}} \frac{\partial f}{\partial z}
$$

Integrating by parts and grouping terms we obtain

$$
H_{\nu}\left[\frac{\lambda}{z^{m-1}} \frac{\partial^{2} f}{\partial z^{2}}+\frac{\rho}{z^{m}} \frac{\partial f}{\partial z} ; s, a, c\right]=\Lambda+\int_{0}^{\infty} f(x, z) L d z,
$$

where

$$
\Lambda=\lambda z^{a-m+1} J_{v}\left(s z^{c}\right) \frac{\partial f}{\partial z}+\left.f(x, z)\left[\rho z^{a-m} J_{v}\left(s z^{c}\right)-\lambda \frac{\partial}{\partial z}\left[z^{a-m+1} J_{v}\left(s z^{c}\right)\right]\right]\right|_{0} ^{\infty},
$$


and

$$
L=\lambda \frac{\partial^{2}}{\partial z^{2}}\left[z^{a-m+1} J_{\nu}\left(s z^{c}\right)\right]-\rho \frac{\partial}{\partial z}\left[z^{a-m} J_{\nu}\left(s z^{c}\right)\right] .
$$

Differentiating and regrouping like terms, and utilizing formulas 3 and 4 in [13, p. 97] we obtain

$$
L=\lambda z^{-m-1}\left[-c^{2} s^{2} z^{2 c+a} J_{v}\left(s z^{c}\right)\right]-\lambda z^{a-m-1} J_{v}\left(s z^{c}\right)\left[a^{2}-c^{2} v^{2}-m(m-1+\rho / \lambda)\right]
$$

with $\rho / \lambda=1-2(m-a)$. Finally, we obtain (12) by substituting $L$ in (14), and by rearranging and utilizing definition (1).

\section{Diffusion of dust from ground-level aerial sources}

Here we consider the problem of transport of a heavy pollutant (dust) from a ground level aerial source within the framework of diffusion theory $[1,7,9]$. We assume the Barenblatt criterion $w / \alpha \nu_{0}<1$ [1] as a sufficient condition in order to use diffusion theory in the present analysis. The equation of turbulent diffusion of a heavy pollutant in a plane parallel flow is expressed as

$$
u \frac{\partial q}{\partial x}-w \frac{\partial q}{\partial z}=\frac{\partial}{\partial y}\left(k_{y} \frac{\partial q}{\partial y}\right)+\frac{\partial}{\partial z}\left(k_{z} \frac{\partial q}{\partial z}\right)
$$

where $q$ is the pollutant concentration, $w$ is the settling velocity of the particle, $k_{y}$ and $k_{z}$ are components of the turbulent exchange coefficients, and $u$ is the wind speed. The $x$-axis points along the wind, the $z$-axis points vertically upward, and the $y$-axis is perpendicular to the $x z$-plane.

Consider the strip of ground $0 \leq x \leq L,-\infty<y<+\infty$ being covered by dust particles. The propagation of dust from this strip will be governed by the equation

$$
u \frac{\partial q}{\partial x}-w \frac{\partial q}{\partial z}=\frac{\partial}{\partial z}\left(k_{z} \frac{\partial q}{\partial z}\right)
$$

Let us assume that $u=u_{1} z^{\mu} ; u_{1}, \mu \in \mathbf{R} ; \mu>0$ and $k_{z}=k_{1} z ; k_{1}, w \in \mathbf{R}$. The lower boundary condition (at $z=0$ ) which ensures a positive flow of dust to the atmosphere at $0 \leq x \leq L$ is

$$
-\left.\left(k_{z} \frac{\partial q}{\partial z}+w q\right)\right|_{z=0}=f(x)
$$

which, in general, is a function of $x$. Physically, $f$ denotes the pollutant flux from the ground surface to the atmosphere. In this statement of the problem $f$ is to 
be determined from the observational data as a function of the weather conditions, properties of the ground surface and of the dust itself. Furthermore, we assume that the turbulent pollutant flux on the underlying surface outside the strip is equal to zero

$$
\left.\left(k_{z} \frac{\partial q}{\partial z}\right)\right|_{z=0}=0 .
$$

Since it is assumed that the strip is the only source of pollutant, it follows that

$$
q(0, z)=0, \quad \lim _{z \rightarrow \infty} q(x, z)=0 .
$$

Solution of the problem: The equation

$$
u_{1} z^{\mu} \frac{\partial q}{\partial x}-w \frac{\partial q}{\partial z}=\frac{\partial}{\partial z}\left(k_{1} z \frac{\partial q}{\partial z}\right)
$$

can be written as

$$
\frac{k_{1}}{z^{-1}} \frac{\partial^{2} q}{\partial z^{2}}+\left(k_{1}+w\right) \frac{\partial q}{\partial z}=u_{1} z^{\mu} \frac{\partial q}{\partial x} .
$$

Applying the generalized Hankel transform to (21) and using the differential property (as applied to the differential operator) with

$$
\begin{gathered}
\overline{f_{\nu}}(x, s ; a, c)=\int_{0}^{\infty} z^{a} f(x, z) J_{v}\left(s z^{c}\right) d z, \\
\nu=-\frac{w}{k_{1}(1+\mu)}, \quad a=\frac{w}{2 k_{1}}=\frac{-v(1+\mu)}{2},
\end{gathered}
$$

$c=(1+\mu) / 2, \lambda=k_{1}$, and $\rho=k_{1}+w$, we have

$$
\Lambda-\lambda c^{2} s^{2} H_{v}\left[z^{\mu} q(x, z) ; s, a, c\right]=u_{1} \frac{d}{d x}\left[H_{v}\left[z^{\mu} q(x, z) ; s, a, c\right]\right],
$$

where

$$
\Lambda=\frac{s^{v} f(x)}{2^{v} \Gamma(v+1)} .
$$

The value of $\Lambda$ is obtained by using the given boundary conditions and the asymptotic expansions of the Bessel functions $J_{v}\left(s z^{c}\right)$ as $z \rightarrow \infty$, as well as their behavior for small values of $z[8,13]$. It is observed that the condition $\rho / \lambda=2 a+1=\left(k_{1}+w\right) / k_{1}$ is satisfied. The solution of the ordinary differential equation (23) for $H_{\nu}[\ldots]$ is

$$
H_{v}\left[z^{\mu} q(x, z) ; s, a, c\right]=\frac{s^{v} e^{-\lambda c^{2} s^{2} x / u_{1}}}{u_{1} 2^{\nu} \Gamma(v+1)} \int_{0}^{x} e^{\lambda c^{2} s^{2} t / u_{1}} f(t) d t .
$$


Now, by an appeal to the inversion formula (2), we get

$$
z^{\mu} q(x, z)=\frac{c z^{2 c-a-1}}{u_{1} 2^{\nu} \Gamma(\nu+1)} \int_{0}^{\infty} s J_{\nu}\left(s z^{c}\right)\left[s^{\nu} e^{-\lambda c^{2} s^{2} x / u_{1}} \int_{0}^{x} e^{\lambda c^{2} s^{2} t / u_{1}} f(t) d t\right] d s,
$$

and hence

$$
q(x, z)=\frac{c z^{-\mu+2 c-a-1}}{u_{1} 2^{\nu} \Gamma(\nu+1)} \int_{0}^{x} f(t)\left[\int_{0}^{\infty} s^{\nu+1} J_{\nu}\left(s z^{c}\right) e^{-\lambda c^{2} s^{2}(x-t) / u_{1}} d s\right] d t .
$$

Evaluating the $s$-integral using Weber's integral formula [8, p. 132]

$$
\int_{0}^{\infty} e^{-a^{2} x^{2}} J_{\nu}(b x) x^{\nu+1} d x=\frac{b^{\nu}}{\left(2 a^{2}\right)^{\nu+1}} e^{-b^{2} / 4 a^{2}}, \quad a, b>0, \operatorname{Re} v>-1,
$$

we obtain

$$
q(x, z)=\frac{\left(u_{1} / k_{1}\right)^{\nu+1} z^{v(1+\mu)}}{(1+\mu)^{2 v+1} u_{1} \Gamma(\nu+1)} \int_{0}^{x} \frac{f(t)}{(x-t)^{v+1}} \exp \left(\frac{-u_{1} z^{\mu+1}}{k_{1}(1+\mu)^{2}(x-t)}\right) d t
$$

with $0 \leq x \leq L$, which provides us with an expression for the pollutant concentration in terms of $f(x)$ - flow of dust into the atmosphere.

\section{Examples}

We consider here some special cases of the solution (29) for a prescribed $f(t)$. Let $f(t)=A_{0} t^{\delta}$ in (29), $A_{0}$ and $\delta$ being constants with $\delta+1>0$. Then we obtain

$$
q(x, z)=\frac{z^{\nu(1+\mu)}\left(u_{1} / k_{1}\right)^{\nu+1} A_{0}}{u_{1} \Gamma(\nu+1)(1+\mu)^{2 v+1}} \int_{0}^{x} \frac{t^{\delta}}{(x-t)^{\nu+1}} \exp \left(\frac{-u_{1} z^{1+\mu}}{k_{1}(1+\mu)^{2}(x-t)}\right) d t .
$$

If we set $K(z)=u_{1} z^{1+\mu} / k_{1}(1+\mu)^{2}$ and $u=K(z) /(x-t)$, then (30) may be written as

$$
q(x, z)=\frac{A_{0}}{\Gamma(\nu+1)(1+\alpha) k_{1}} \int_{K(z) / x}^{\infty}\left[x-\frac{K(z)}{u}\right]^{\delta} \exp (-u) u^{\nu-1} d u .
$$

By an appeal to the integral formula (6) in [10, p. 324], we get

$$
q(x, z)=\frac{A_{0} x^{\delta} \Gamma(\delta+1)}{\Gamma(\nu+1)(1+\mu) k_{1}}(K(z) / x)^{\nu} e^{-K(z) / x} \psi(\delta+1, \nu+1 ; K(z) / x),
$$


where $\psi(\alpha, \gamma ; z)$ is the confluent hypergeometric function of the second kind [8] defined as

$$
\psi(\alpha, \gamma ; z)=\frac{1}{\Gamma(\alpha)} \int_{0}^{\infty} e^{-z t} t^{\alpha-1}(1+t)^{\gamma-\alpha-1} d t, \quad \operatorname{Re} \alpha, \quad \operatorname{Re} z>0 .
$$

The expression for the pollutant concentration given by (32) is a general result and a number of special cases can be derived from it.

Let $\delta=(\nu-1) / 2$. Then by virtue of the formula [8]

$$
K_{\nu}(z)=\sqrt{\pi}(2 z)^{\nu} e^{-z} \psi(\nu+1 / 2,2 v+1 ; 2 z),
$$

and (32) reduces to

$$
q(x, z)=\frac{A_{0} \Gamma(\nu / 2+1 / 2) x^{(\nu-1) / 2}}{k_{1} \Gamma(\nu+1)(1+\mu) \sqrt{\pi}}(K(z) / x)^{\nu / 2} e^{-K(z) / 2 x} K_{\nu / 2}(K(z) / 2 x),
$$

where $K_{\rho}(z)$ is the modified Bessel function (MacDonald function) $[8,13]$.

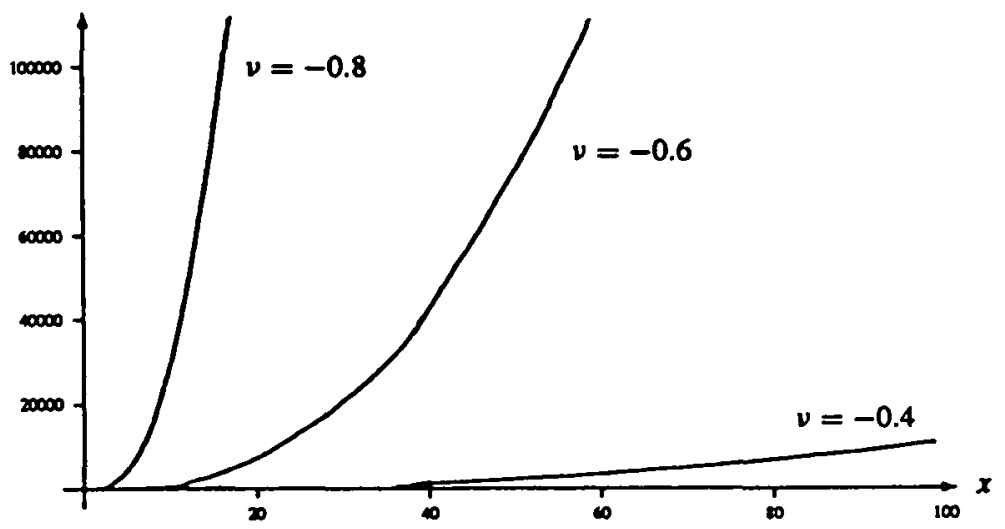

FIGURE 1. Ground level concentration $q(x, 0)$ for $\delta=2$, and $\nu=-0.8,-0.6,-0.4$.

If we set $\delta=0, \mu=n \in \mathbf{N}$ in (32), that is, the positive flow of dust to the atmosphere in $0 \leq x \leq L$ is assumed to be constant $A_{0}$, then we obtain the well-known result $[7,9]$

$$
q(x, z)=\frac{A_{0}}{\Gamma(\nu+1)(1+n) k_{1}} \Gamma(\nu, K(z) / x),
$$

where $\Gamma(a, x)$ is the complementary incomplete gamma function [8] defined as

$$
\Gamma(a, x)=\int_{x}^{\infty} e^{-t} t^{a-1} d t
$$




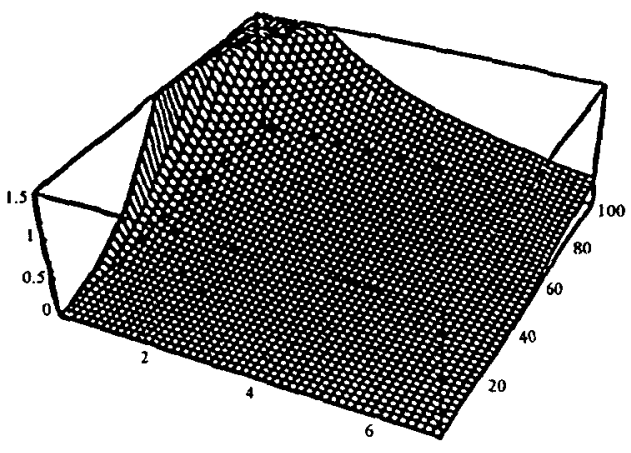

(a) $v=-0.2$

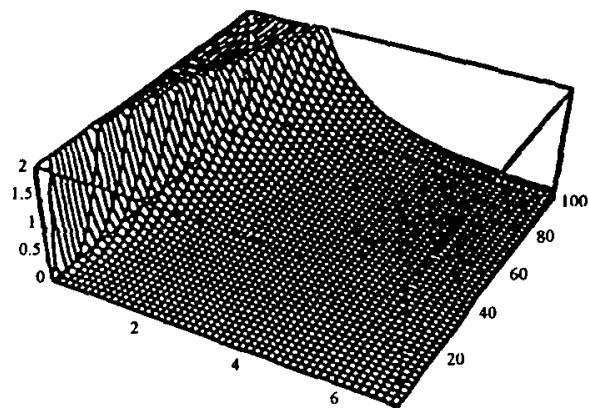

(b) $v=-0.8$

\section{(c) $v=-0.001$}

FIGURE 2. Pollutant concentration $q(x, z)$ for $x \in[0,100], z \in[0,7] . A_{0}=10^{-4}, \mu=k_{1}=u_{1}=1$, $\delta=2$, and $v=-0.8,-0.2,-0.001$.

with $\nu$ as in (22). Here we have used the formulae $\psi(1-a, 1-a ; x)=e^{x} \Gamma(a, x)$, and $\psi(a, b ; x)=x^{1-b} \psi(1+a-b, 2-b ; x)$.

As another example, let us consider $f(t)=A_{0} t^{\delta} e^{-\gamma t}$, where $A_{0}, \delta$ and $\gamma$ are positive constants. Then, from (29), the pollutant concentration becomes

$$
q(x, z)=\frac{z^{\nu(1+\mu)}\left(u_{1} / k_{1}\right)^{\nu+1} A_{0}}{u_{1} \Gamma(\nu+1)(1+\mu)^{2 \nu+1}} \int_{0}^{x} \frac{t^{\delta} e^{-\gamma t}}{(x-t)^{\nu+1}} \exp \left(\frac{-u_{1} z^{1+\mu}}{k_{1}(1+\mu)^{2}(x-t)}\right) d t
$$

which can be rewritten as

$$
\begin{aligned}
q(x, z) & =\frac{A_{0} x^{\delta} e^{-\gamma x}}{\Gamma(\nu+1)(1+\mu) k_{1}} \int_{K(z) / x}^{\infty}\left[u-\frac{K(z)}{x}\right]^{\delta} u^{\nu-\delta-1} e^{-u} e^{\gamma K(z) / u} d u \\
& =\frac{A_{0} x^{\delta} e^{-\gamma x}}{\Gamma(\nu+1)(1+\mu) k_{1}} \sum_{r=0}^{\infty} \frac{\gamma^{r}}{r !} \int_{K(z) / x}^{\infty}\left[u-\frac{K(z)}{x}\right]^{\delta} u^{\nu-\delta-1} e^{-u}\left(\frac{K(z)}{u}\right)^{r} d u \\
& =\frac{A_{0} x^{\delta} e^{-\gamma x}}{\Gamma(\nu+1)(1+\mu) k_{1}} \sum_{r=0}^{\infty} \frac{(\gamma K(z))^{r}}{r !} \int_{K(z) / x}^{\infty}\left[u-\frac{K(z)}{x}\right]^{\delta} u^{\nu-\delta-r-1} e^{-u} d u
\end{aligned}
$$




$$
\begin{aligned}
= & \frac{A_{0} x^{\delta} e^{-\gamma x}}{\Gamma(\nu+1)(1+\mu) k_{1}} \Gamma(\delta+1) \\
& \times \sum_{r=0}^{\infty} \frac{(\gamma K(z))^{r}}{r !}(K(z) / x)^{\nu-r} e^{-K(z) / x} \psi(\delta+1, v-r+1 ; K(z) / x) .
\end{aligned}
$$

If we set $\gamma=0$ in this result, it reduces to (32), while $\gamma=\delta=0$ leads to (36).

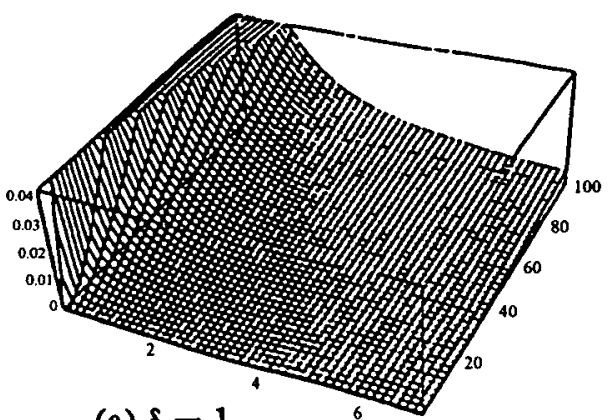

(a) $\delta=1$

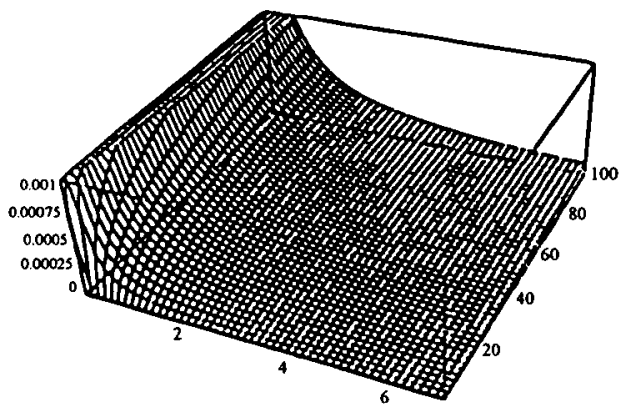

(b) $\delta=0$

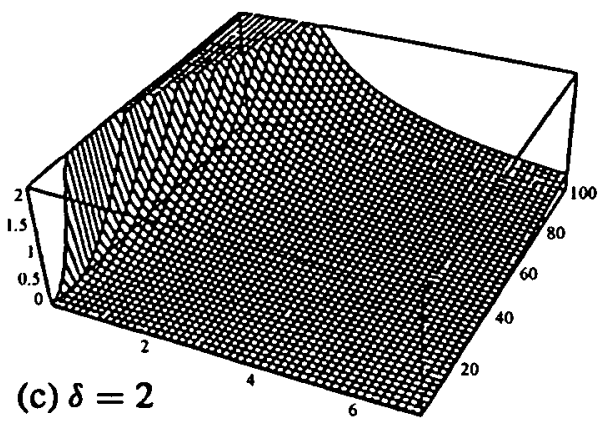

FIGURE 3. Pollutant concentration $q(x, z)$ for $\nu=-0.4$, and $\delta=2,1,0$.

\section{Discussion of the results}

We have defined a generalized form of the Hankel transform and obtained some of its properties. This transform can be used to solve some partial differential equations. As an example we have considered the problem of the transport of a heavy pollutant (dust) from ground level aerial sources. Pollutant concentration $q(x, z)$ in the strip $0 \leq x \leq L$ is derived, and expressed in terms of the flow of dust to the atmosphere $f(x)$. Two separate models have been considered; when the flow of dust to the atmosphere is represented by a power function, and, in the second case, as a product of a power and an exponential function. The results are expressed in terms of known special functions $[6,8,13]$. 


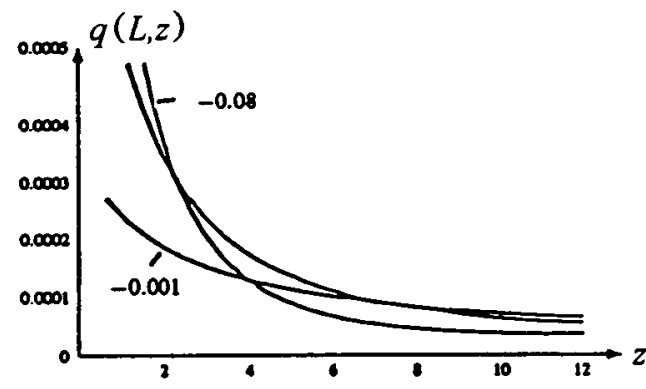

(a) $\delta=0$

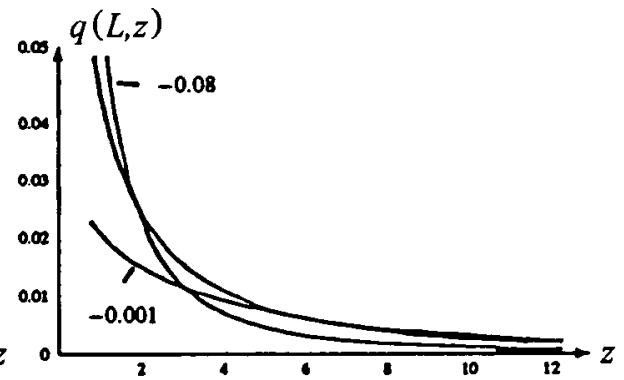

(b) $\delta=1$

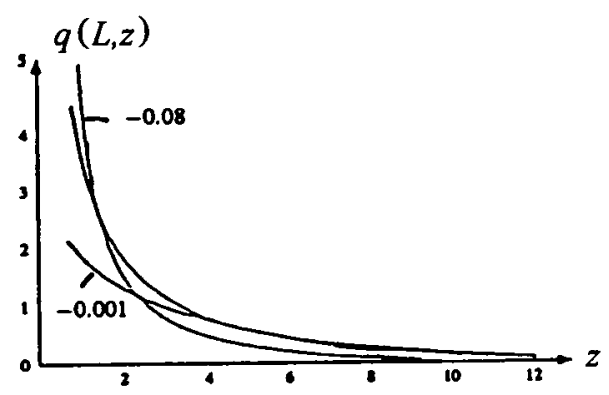

(c) $\delta=2$

FIGURE 4. Pollutant concentration $q(L, z)$ for $z \in[1,12]$.

The general result (29) can yield the pollutant concentration at the edge of the strip $x=L$, that is, $q(L, z)$. Using an integral representation of the solution of an analogous problem with arbitrary initial pollutant distribution obtained by Berlyand [3], together with $q(L, z)$, one can obtain the pollutant concentration $q(x, z)$ for $x>L$. We propose to discuss the details in a subsequent paper. In this case $(x>L)$ one can obtain the ground level concentration $q(x, 0)$, and the corresponding results for lighter particles.

The following figures represent the pollutant concentration $q(x, z)$ in the strip $0 \leq x \leq L$ for various values of $\delta$ and $\nu$ - result (32). These figures are only indicative of the relative dependence of $q(x, z)$ on the parameters involved, and are not based on actual meteorological data. Figure 1 shows the ground level concentration $q(x, 0)$ for different values of $v$, where we note that larger negative values of $v$ (that is, larger settling velocities) result in higher concentrations at the ground level. Figure 2 represents the pollutant concentration $q(x, z)$ for $x \in[0,100]$, and $z \in[0,7]$. Here we have set $A_{0}=10^{-4}, \mu=k_{1}=u_{1}=1, \delta=2$, and $\nu=-0.8,-0.2$, and -0.001 . As the settling velocities decrease there is more pollutant concentration in the atmosphere. In Figure 3, $q(x, z)$ is shown for a fixed value of $v=-0.4$ while 
$\delta=2,1$, and 0 . Here again we have $A_{0}=10^{-4}, \mu=k_{1}=u_{1}=1,0 \leq x \leq L$, $0 \leq z \leq 7$, and $L=100$. As expected, $q(x, z)$ increases with both $x$ and $\delta$. Finally, Figure 4 represents $q(L, z)$ for $1 \leq z \leq 12$, where $\nu=-0.001,-0.4$, and -0.8 , and $\delta=0,1$, and 2. This figure indicates that smaller negative values of $v$ (that is, smaller settling velocities) result in higher concentrations at high altitudes.

\section{Acknowledgement}

The authors are grateful to Professor J. Harper and the referees for their suggestions and useful comments. This work was undertaken whilst one of the authors SK was visiting the Department of Mathematics and Computer Science at Kuwait University.

\section{References}

[1] G. I. Barenblatt, "Motion particles in turbulent flow occupying a half space or a flat duct of finite depth”, Prikl. Mat. i Mekh. 17 (1953) 261-274.

[2] G. I. Barenblatt, "Motion of suspended particles in turbulent flow", Prikl. Mat. i Mekh. 19 (1955) 61-88.

[3] M. Ye. Berlyand, Sovremennyye problemy atmosfernoy diffuzii i zagryazneniya atmosfery (Problems of atmospheric diffusion and pollution) (Gidrometeorizdat Press, Leningrad, 1975).

[4] E. K. Byutner, Dinamika pripoverkhostnogo sloya vozdukha (Dynamics of the ground-level air layer) (Gidrometeorizdat Press, Leningrad, 1978).

[5] B. Davies, Integral Transforms and Their Applications (Springer-Verlag, New York, 1978).

[6] A. Erdelyi, Higher Transcendental Functions, 3 volumes (McGraw-Hill, New York, 1953-1954).

[7] S. L. Kalla and E. Urribarri, "Hankel transform to the diffusion of dust problem", in Proc. 5-th. Int. Colloquium Diff. Eq. (Plovdiv, Bulgaria, 1994), 173-180.

[8] N. N. Lebedev, Special Functions and their Applications (Prentice-Hall, Englewood Cliffs, N.J., 1965).

[9] R. I. Onikul and L. G. Khurshudyan, "The diffusion of dust from its ground-level aerial sources", Fluid Mechanics-Soviet Research 13 (1984) 64-74.

[10] A. P. Prudnikov, Yu. A. Brychkov and O. I. Marichev, Integrals and Series (Gordon and Breach Science Publishers, Reading, U.K., 1992).

[11] I. N. Sneddon, The use of Integral Transforms (McGraw-Hill, New York, 1972).

[12] C. J. Tranter, Integral Transforms in Mathematical Physics (Chapman and Hall, London, 1971).

[13] G. N. Watson, A Treatise on the theory of Bessel functions (Cambridge Univ. Press, London, 1980). 